\title{
Development of autoimmune diabetes with severe diabetic ketoacidosis and immune-related thyroiditis secondary to durvalumab: a case report
}

\author{
Ana Rita Lopes ${ }^{1,2}$, Alessandro Russo ${ }^{1,3}$, Andrew Y. Li $^{4}$, Michael G. McCusker ${ }^{1}$, Jeffrey Myles Kroopnick ${ }^{5}$, \\ Katherine Scilla ${ }^{1}$, Ranee Mehra ${ }^{1}$, Christian Rolfo ${ }^{1}$ \\ ${ }^{1}$ Marlene and Stewart Greenebaum Comprehensive Cancer Center, University of Maryland School of Medicine, Baltimore, MD, USA; ${ }^{2}$ Portuguese \\ Institute of Oncology (IPO), Porto, Portugal; ${ }^{3}$ Medical Oncology Unit, A.O. Papardo, Messina, Italy; ${ }^{4}$ Department of Medicine, Division of General \\ Internal Medicine, University of Maryland Medical Center, Baltimore, MD, USA; ${ }^{5}$ University of Maryland Center for Diabetes and Endocrinology, \\ University of Maryland Medical Center Midtown Campus, Baltimore, MD, USA \\ Correspondence to: Prof. Dr. Christian Rolfo, MD, PhD, MBA, Dr.h.c. Director of Thoracic Medical Oncology and Early Clinical Trials at University \\ of Maryland Medical Center, Greenebaum Comprehensive Cancer Center, 22 S Greene Street Rm. N9E08, Baltimore, MD 21201 , USA. \\ Email: christian.rolfo@umm.edu.
}

\begin{abstract}
Immune-mediated endocrinopathies are among the most frequent immune-related adverse events (irAEs) with immune checkpoint inhibitors (ICIs) targeting programmed death-ligand 1 (PDL1)/PD-1. However, the development of auto-immune diabetes is an uncommon event during $\mathrm{PD}(\mathrm{L})$ 1 blockade, either as monotherapy or in combination therapy. Here we report a case of a 75 -year-old male with a mediastinal recurrence from a stage IA squamous cell carcinoma of the lung previously treated with stereotactic body radiotherapy (SBRT) who early developed a severe diabetic ketoacidosis (DKA) caused by new-onset auto-immune diabetes, with positive glutamic acid decarboxylase (GAD65) autoantibodies, during durvalumab consolidation therapy after concurrent chemoradiation. The patient had no personal or family history of diabetes or auto-immune diseases and was admitted after the second cycle of durvalumab to the intensive care unit (ICU) with severe DKA. During his hospitalization, insulin and fluid therapy were started and the patient had a favorable clinical course. Durvalumab treatment was interrupted and thyroiditis was verified during follow-up, without anti-thyroid antibodies, that progressed to subsequent hypothyroidism with need of thyroid hormone replacement therapy. This case highlights the rare irAE of autoimmune type 1 diabetes during anti-PD(L)-1 therapy, which can be life-threatening and requires adequate patient education and prompt medical treatment within a multidisciplinary team, including endocrinology and emergency medicine. Besides its low incidence, this case show how irAE must be taken in account about decision of ICI treatment, especially in curative setting, as they can be potentially fatal and impair overall survival. Furthermore, as reported in the present case, multiple endocrine irAEs can occur in the same patient either simultaneously or sequentially, suggesting that active surveillance is needed in those who develop endocrinopathies as a result of ICI treatment. Immune-mediated endocrinopathies are generally irreversible and cause life-long morbidity, which must be taken into consideration when deciding on further lines of treatment.
\end{abstract}

Keywords: Autoimmune polyendocrinopathy; auto-immune diabetes; thyroiditis; durvalumab; case report

Submitted Mar 09, 2020. Accepted for publication Jun 09, 2020.

doi: $10.21037 /$ tlcr-20-408

View this article at: http://dx.doi.org/10.21037/tlcr-20-408 


\section{Introduction}

Durvalumab is a PD-L1 blocking antibody which is FDA approved as consolidation therapy in patients with unresectable stage III non-small cell lung cancer (NSCLC), whose disease has not progressed after concurrent chemoradiation therapy. This approval is based on the PACIFIC trial, which showed a statistically significant improvement in median progression-free survival (16.8 vs. 5.6 months) and longer overall survival (36-month overall survival rate $57.0 \%$ vs. $43.5 \%)$ in comparison with placebo $(1,2)$.

In the PACIFIC trial, immune-related adverse events (irAEs) of any grade were reported in $24.2 \%$ of patients treated with durvalumab, of which $3.4 \%$ were grade 3 or 4. The most frequent endocrinopathies of any grade were hypothyroidism (11.6\%) and hyperthyroidism (6.3\%), although one patient also developed type 1 diabetes mellitus $(0.2 \%)$. Awareness of the potential irAEs by medical professionals and patients is important for their early detection and treatment. Management of irAEs can include withholding immunotherapy or initiating hormone replacement or immunosuppressive treatments, thereby preventing an unfavorable clinical evolution that may compromise patients' overall survival and quality of life. Here, we report a case of a new onset auto-immune diabetes with life-threatening DKA after the second cycle of durvalumab in a patient who completed concurrent chemoradiation from mediastinal disease which was recurrent from a previously treated Stage IA squamous cell carcinoma of the lung. This case is also complicated by the development of thyroiditis after suspension of durvalumab. With this case report, the authors want to highlight the potential life-threatening presentation of autoimmune diabetes associated with ICIs and emphasizes that, although rare, the irAEs must be taken in account about decision of treatment, especially nowadays that ICIs are investigated even more in curative setting, where the severity and lifelong morbidity of irAE are of special importance and may limit further lines of treatment. We present the following case in accordance with the CARE Reporting Checklist (3). Written informed consent was obtained from the patient for publication of this case report and any accompanying images.

We present the following case in accordance with the CARE Reporting Checklist (available at http://dx.doi. org/10.21037/tlcr-20-408).

\section{Case presentation}

A 75 -year-old Caucasian male with history of former tobacco use (40 pack years) and an Eastern Cooperative Oncology Group (ECOG) performance status of 1 was diagnosed in October 2017 with squamous cell carcinoma (SCC) of right lower lobe of lung [cT1aN0M0; stage IA (AJCC $7^{\text {th }}$ edition); PD-L1 expression unknown]. He had a history of chronic obstructive pulmonary disease, hypertension, dyslipidemia, pulmonary embolism on therapeutic anticoagulation and benign prostatic hyperplasia, and had no personal or family history of autoimmune or endocrine diseases, including diabetes.

The patient refused surgery and was initially treated with SBRT at a total dose of 50 Gy over four fractions. After 9 months, he developed an isolated recurrence in mediastinal lymph nodes (station 7). After locoregional recurrence treated with salvage chemo-radiation therapy, an individualized multidisciplinary discussion is recommended as durvalumab is now the standard care for de novo stage III NSCLC, although there is no data to support its use for this indication (4). After a thoracic oncology multidisciplinary discussion, concurrent chemoradiation with weekly carboplatin/paclitaxel for 6 weeks was proposed, followed by consolidation therapy with durvalumab at the dose of $10 \mathrm{mg} / \mathrm{kg}$ intravenously every 2 weeks. Durvalumab treatment started on February 14, 2019, but cycle two was delayed three weeks because of insurance issues. On Day 12 of Cycle 2, he presented at the emergency department with 2-day history of dyspnea, generalized weakness, dizziness, dysphagia, nausea/vomiting, and diarrhea (Figure 1). He denied fever, chills, cough, chest pain, abdominal pain, urinary symptoms, headache or other complaints.

On admission, the patient was alert and oriented, hemodynamic stable with blood pressure of $104 / 53$ $\mathrm{mmHg}$ and tachycardia (129 bpm), tachypnea (25 breaths per minute with oxygen saturation of $97 \%$ on room air), without fever $\left(36.4^{\circ} \mathrm{C}\right)$, dry skin with oral mucosa moist, irregular heart rate with tachycardic S1 and S2 and no murmur heard. The remainder of the physical exam was unremarkable. Electrocardiogram revealed atrial fibrillation with rapid ventricular response and laboratory studies were 


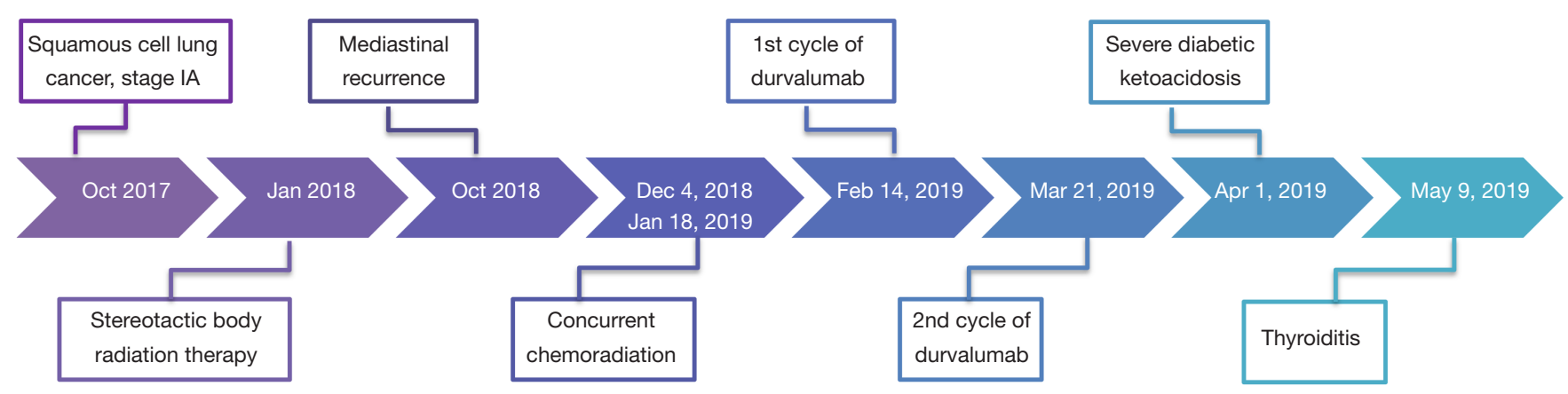

Figure 1 Flowchart of oncologic clinical history, treatments performed and immune-related adverse events.

consistent with severe DKA, with electrolyte disorders (hyperkalemia and hypophosphatemia) and acute kidney injury, with an estimated glomerular filtration rate (GFR) of $14.54 \mathrm{~mL} / \mathrm{min} / 1.73 \mathrm{~m}^{2}$ in comparison with a baseline GFR of $66.60 \mathrm{~mL} / \mathrm{min} / 1.73 \mathrm{~m}^{2}$, using the Modification of Diet in Renal Disease (MDRD) formula (Table 1).

The patient was admitted to the ICU and was treated with intravenous hydration with sodium bicarbonate sodium and calcium gluconate, intravenous insulin therapy, as well as esmolol and amiodarone for control of atrial fibrillation. During the hospitalization, the diagnostic work-up confirmed an auto-immune diabetes with positive glutamic acid decarboxylase (GAD65) autoantibodies $(64 \mathrm{IU} / \mathrm{mL}$, normal $<5 \mathrm{IU} / \mathrm{mL}$ ), negative anti-islet cell antibodies and glycated hemoglobin (HbA1c) of $7.5 \%$ (normal range, 4.3-5.6\%). The thyroid function was normal with TSH $0.40 \mathrm{UIU} / \mathrm{mL}$ (normal range, 0.35-5.50), cortisol was $26.9 \mathrm{mcg} / \mathrm{dL}$ (normal range, 10.0-25.0) and the blood cultures and other sepsis screening exams were all negative.

The patient had a favorable clinical course during the hospitalization and insulin therapy was switched to subcutaneous after 24 hours. After treatment, the heart rhythm was converted to normal sinus rhythm and the echocardiogram was normal, with left ventricular ejection fraction (LVEF) estimated of 55-60\%. He was discharged with insulin detemir 20 units twice daily and preprandial insulin aspart, with improvement of metabolic, electrolytic and renal dysfunction (glucose $94 \mathrm{mg} / \mathrm{dL}$, urea $43 \mathrm{mg} / \mathrm{dL}$, creatinine $1.30 \mathrm{mg} / \mathrm{dL}$, sodium $143 \mathrm{mmol} / \mathrm{L}$, potassium $3.7 \mathrm{mmol} / \mathrm{L}$, calcium $8.6 \mathrm{mg} / \mathrm{dL}$, magnesium $1.9 \mathrm{mg} / \mathrm{dL}$ and phosphorous $2.7 \mathrm{mg} / \mathrm{dL}$ ).

The treatment with durvalumab was discontinued due to grade 4 auto-immune diabetes. During follow-up visits, the patient presented with moderate glycemic control and need for adjustment of insulin therapy (Figure 2). During treatment, the patient also experienced xerostomia grade 1 and no other side effects.

Approximately two months after the last dose of durvalumab, thyroiditis was diagnosed with normal thyroid autoantibodies (Figure 2) and intact hypothalamic, pituitary and adrenal axis, with cortisol of $12.3 \mathrm{mcg} / \mathrm{dL}$ (normal range, 10.0-25.0) and ACTH $15 \mathrm{pg} / \mathrm{mL}$ (normal range, $6-50)$. There was a calcified nodule in the right lobe of thyroid, but without abnormally increased ${ }^{18} \mathrm{~F}-\mathrm{FDG}$ uptake on PET/CT. No therapy was administered due to asymptomatic thyrotoxicosis and it progressed to subsequent hypothyroidism, with need for thyroid hormone replacement therapy.

During surveillance, there was progression of disease with a metabolically active right level $2 \mathrm{~A}$ cervical lymph node on PET/CT measuring up to $5 \mathrm{~mm}$ (SUVmax $=18$ ); the soft tissues of the nasopharynx, oropharynx, hypopharynx and larynx were within normal limits and no other signs of distant metastasis. Fine needle aspiration cytology was positive for malignant cells compatible with metastatic squamous cell carcinoma of the lung, outside the field of radiation.

\section{Discussion}

ICIs can trigger autoimmune reactions in any organ system and endocrine irAEs are the most common, with an incidence of 4-30\% of any grade in clinical trials (5). These immune-mediated endocrinopathies can affect the thyroid, pituitary, adrenal glands and pancreatic cells, causing hypothyroidism, hyperthyroidism, hypophysitis, primary adrenal insufficiency and type 1 diabetes mellitus. Thyroid 
Table 1 Laboratory analysis results on admission to emergency department

\begin{tabular}{|c|c|c|}
\hline Test & Value & Reference range \\
\hline \multicolumn{3}{|l|}{ Blood } \\
\hline Hemoglobin (g/dL) & 12.4 & $12.8-16.6$ \\
\hline White blood cells $\left(\times 10^{3} / \mathrm{mm}^{3}\right)$ & 15.1 & $3.9-9.5$ \\
\hline Neutrophils (\%) & 92 & $47.1-77.6$ \\
\hline Platelets $\left(\times 10^{3} / \mathrm{mm}^{3}\right)$ & 341 & $140-366$ \\
\hline Glucose (mg/dL) & 1532 & $70-99$ \\
\hline Urea (mg/dL) & 90 & $7-25$ \\
\hline Creatinine (mg/dL) & 4.26 & $0.7-1.3$ \\
\hline Sodium (mmol/L) & 122 & $136-145$ \\
\hline Potassium (mmol/L) & 6.8 & $3.5-5.1$ \\
\hline Chloride (mmol/L) & 82 & $98-107$ \\
\hline Calcium (mg/dL) & 9.6 & $8.6-10.3$ \\
\hline Phosphorous (mg/dL) & 1.9 & $2.3-4.6$ \\
\hline Magnesium (mg/dL) & 3.0 & $1.9-2.7$ \\
\hline Albumin (g/dL) & 3.8 & $3.5-5.7$ \\
\hline Ketones & High & Negative \\
\hline AST (U/L) & 20 & $13-39$ \\
\hline ALT (U/L) & 35 & $7-52$ \\
\hline Alkaline phosphatase (U/L) & 76 & $34-104$ \\
\hline Total bilirubin (mg/dL) & 1.6 & $0.3-1.0$ \\
\hline Lipase (U/L) & 130 & $11-82$ \\
\hline \multicolumn{3}{|l|}{ Arterial blood gas } \\
\hline $\mathrm{pH}$ & 6.99 & $7.35-7.45$ \\
\hline $\mathrm{pO}_{2}(\mathrm{mmHg})$ & 121 & $75-100$ \\
\hline $\mathrm{pCO}_{2}(\mathrm{mmHg})$ & 7 & $35-45$ \\
\hline $\mathrm{HCO}_{3}(\mathrm{mmol} / \mathrm{L})$ & 2 & $22-29$ \\
\hline Base deficit (mmol/L) & 27.3 & -2.0 to 3.0 \\
\hline Anion gap (mmol/L) & 33 & $3-11$ \\
\hline Lactic (mmol/L) & 4.8 & $0.5-2.2$ \\
\hline \multicolumn{3}{|l|}{ Urinalysis } \\
\hline Glucose (mg/dL) & $\geq 1,000$ & Negative \\
\hline Ketones (mg/dL) & 15 & $<0.8$ \\
\hline
\end{tabular}

disorders are the most common endocrine irAEs, with a reported overall incidence of $4.0-15.0 \%$ for hypothyroidism and $3.2-9.9 \%$ for hyperthyroidism (5), but the incidence is also influenced by which ICI is administered. In a meta-analysis of 38 randomized clinical trials comprising 7,551 patients treated with $\mathrm{PD}-\mathrm{L} 1 / \mathrm{PD}-1$ inhibitors (pembrolizumab, nivolumab or atezolizumab) or CTLA4 (cytotoxic T-lymphocyte-associated protein-4) inhibitors (ipilimumab), treatment with PD-L1/PD-1 inhibitors or combination therapy of PD-L1/PD-1 plus CTLA4 inhibitors was significantly more likely to cause any grade of hypothyroidism in comparison with ipilimumab monotherapy, with an odds ratio (OR) 1.89 (95\% CI: $1.17-$ 3.05) and OR 3.81 (95\% CI: 2.10-6.91), respectively (6). Concerning hyperthyroidism, PD-L1/PD-1 plus CTLA4 combination therapy was also associated with higher risk in comparison with ipilimumab (OR 4.27; 95\% CI: 2.05-8.90), which was not statistically significant for PDL1/PD-1 inhibitors. The risk of hyperthyroidism was also significantly greater with PD-1 inhibitors than with PD-L1 inhibitors (OR 5.36; 95\% CI: 2.04-14.08).

Hypophysitis is less common, with an observed incidence of $0.6-7.7 \%$ (5) and the observed incidence was greatest with combination therapy of PD-L1/PD-1 plus CTLA4 inhibitors $(6.4 \%)$, followed by treatment with CTLA4 inhibitors (3.2\%), PD-1 inhibitors (0.4\%) and less than $0.1 \%$ for PD-L1 inhibitors (6). The incidence of all grades of primary adrenal insufficiency and auto-immune diabetes is much less and, in the meta-analysis mentioned above, only 13 cases of any-grade of auto-immune diabetes were reported $(0.2 \%)$, with 6 patients experiencing grade 3 or higher $(0.1 \%)$ and appears to be more common with PD-1 and PD-L1 blockade as all the cases were associated with PD-1 inhibitors, except in one case treated with ipilimumab (6).

Durvalumab can also cause several immune-mediated endocrinopathies and, in clinical studies enrolling 1,889 patients, hypothyroidism occurred in $11 \%$ of patients, hyperthyroidism in $7 \%$, thyroiditis in $0.9 \%$ and type 1 diabetes mellitus $<0.1 \%$, with a median time to onset for diabetes of 1.4 months (7). As in our case report, the association of auto-immune diabetes presenting with DKA and simultaneous thyroiditis during durvalumab 

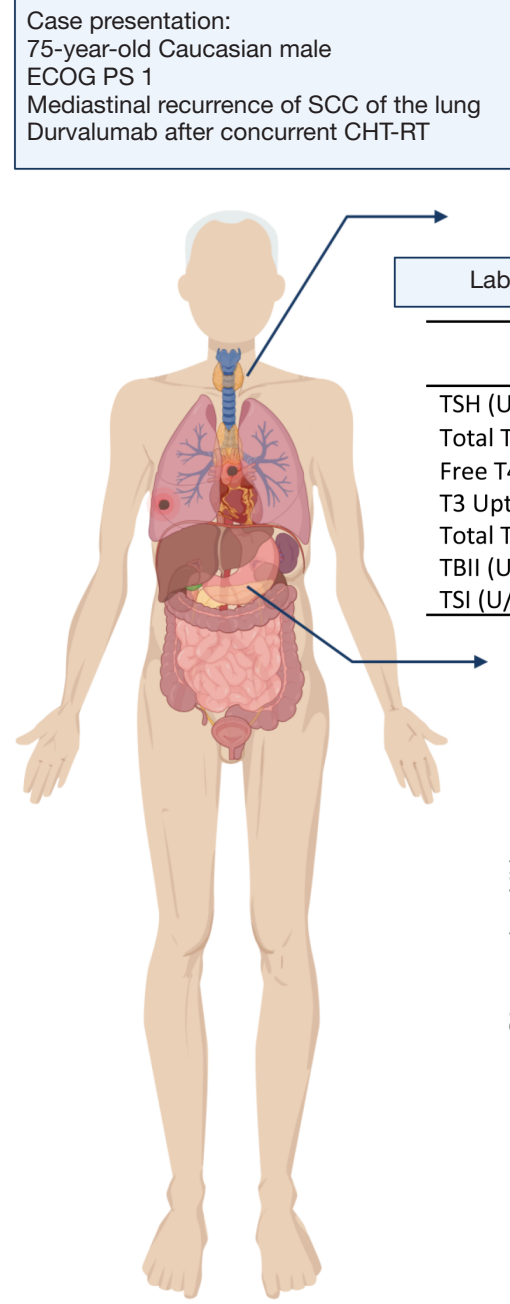

Thyrotoxicosis followed by hypothyroidism

\begin{tabular}{lcccc}
\multicolumn{4}{c}{ Laboratory analysis results of thyroid function during durvalumab treatment and follow-up } \\
& $\begin{array}{c}\mathbf{2 0 1 9 . 2 . 1 4} \\
\text { (Cycle 1) }\end{array}$ & $\mathbf{2 0 1 9 . 5 . 2 1}$ & $\mathbf{2 0 1 9 . 7 . 2 3}$ & $\begin{array}{c}\text { Reference } \\
\text { range }\end{array}$ \\
\hline TSH (UIU/L) & 2.15 & $<0.01$ & 31.24 & $0.35-5.50$ \\
Total T4 (mcg/dL) & & 13.5 & & $4.6-11.0$ \\
Free T4 (ng/dL) & 1.1 & 2.0 & 0.2 & $0.6-1.6$ \\
T3 Uptake (\%) & & 36 & & $22-35$ \\
Total T3 (nmol/L) & 1.67 & & $0.87-1.87$ \\
TBII (U/L) & $<6.0$ & $<1.75$ \\
TSI (U/L) & 89 & & $<140$ \\
\hline
\end{tabular}

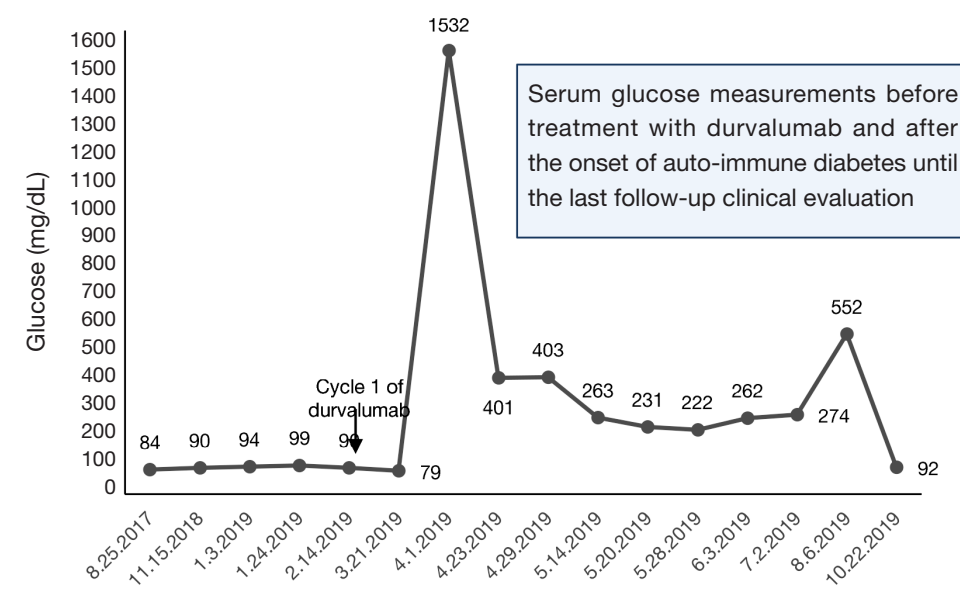

Figure 2 Summary of major clinico-pathological characteristics and adverse events. Serum glucose measurements before treatment with durvalumab and after the onset of auto-immune diabetes until the last follow-up clinical evaluation are reported at the bottom of the figure. In the upper part are included the major laboratory analysis results of thyroid function during durvalumab treatment and in follow-up. T3, triiodothyronine; T4, thyroxine; TSH, thyroid stimulating hormone; TBII, TSH-binding inhibitor immunoglobulin; TSI, thyroid stimulating immunoglobulin antibody.

treatment has already been reported in a 55 -year-old male with urothelial high-grade non-muscle-invasive bladder cancer (8).

The incidence of autoimmune diabetes is expected to increase with the use of ICIs in clinical practice for treatment of many cancers. A total of 283 cases of new-onset diabetes mellitus from 2014 to April 2018 following treatment with ICIs were reported in VigiBase, the World Health Organization's database of individual adverse drug reactions (9). Overall, the majority of the patients with auto-immune diabetes presented with DKA (50.2\%) and the onset of diabetes ranged from 5 to 790 days after the first dose of ICIs (median 116 days), but $9 \%$ of the patients developed diabetes even 3 months or more after stopping ICI treatment.

In a systematic review of autoimmune diabetes related to ICIs, 91 case reports were analyzed. There was a male predominance (60\%), mean age of 61 years (range, $22-84$ years) and most of the patients were treated with PD-1 or PD- 
L1 inhibitors as monotherapy (79\%) or in association with anti-CTLA4 (15\%) (10). The presentation was generally severe and $71 \%$ of patients presented with DKA, with a median presenting serum glucose measurement of $565 \mathrm{mg} /$ $\mathrm{dL}$ (range, 209-1,211). The autoantibody analysis was most commonly positive for GAD65 (51\%), followed by insulin (26\%), insulinoma-associated antigen-2 (IA-2) in $18 \%$, islet-cell antibodies (ICA) in 13\% and zinc transporter 8 ( $\mathrm{ZnT} 8)$ in 4\%. However, not all these autoantibodies were systematically analyzed, providing bias in the study. Concerning other concomitant endocrine irAEs, thyroid dysfunction (thyroiditis, primary hypo- or hyperthyroidism) was the most frequent with an incidence of $24 \%$ and this association of auto-immune diabetes and thyroiditis was also observed in our case report.

Time of onset for immune-mediated endocrinopathies is also dependent on ICIs and the grade 3-4 irAEs occur approximately at $12.1(2.9-17.0)$ and $28.6(19.1-38.1)$ weeks after the first dose of PD-1 plus CTLA-4 or PD-1 (nivolumab) inhibitors, respectively (11), although they can manifest later and even after interruption of treatment, highlighting the importance of continuous follow-up concerning irAEs for several months. Early-onset diabetes mellitus (after one or two cycles) has been observed in all treatment regimens but, on average, diabetes mellitus was diagnosed after 4.5 cycles (range, 1-17); an earlier onset was noted for those receiving anti-PD-1 and anti-CTLA4 combination therapy (2.7 cycles; range, 1-5), patients presenting with DKA (4 cycles) and those with GAD65-positivity (3.1 cycles; range, 1-16) (10). Diabetic ketoacidosis seems a common clinical presentation at diagnosis and is reported in $69 \%$ of cases (12).

In our case report, an increased lipase level of grade 2 was verified on emergency admission, which was consistent with findings in a systematic review of 91 cases (10), in which $52 \%$ of patients had an elevated lipase measurement which was thought to reflect pancreatic inflammation. Another retrospective study has described evidence of pancreatitis in the peridiagnosis period in $42 \%$ of the patients diagnosed with auto-immune diabetes (13). This elevation of lipase can be associated with immune-related pancreatitis but, in a study with 21 patients treated with anti-PD-1 or anti-PD-L1, 71\% had an asymptomatic elevation of lipase without radiographic abnormalities of the pancreas, that was not considered as clinically significant and the treatment was continued, without later diagnosis of new-onset diabetes or other complications (14). Concerning $\mathrm{HbA1c}$, it has generally been reported as $6-7.95 \%$ (range, 5.4-11.4\%), suggesting that some degree of hyperglycemia or significant hyperglycemia during a shorter period had been present prior to the acute presentation $(10,13)$.

The physiopathology of auto-immune diabetes associated with ICIs is unknown and auto-antibodies are seen only in $40-51 \%$ of cases $(10,13)$. Its principal association with PD-1 and PD-L1 inhibitors may be related to the expression of PD-L1 on pancreatic beta cells and pancreatic islets. Indeed, a deficiency in the ability to upregulate PD-1 and use of the PD-1/PD-L1 pathway has been observed in regulatory $\mathrm{T}$ cells (Tregs) from patients with type 1 diabetes (10). ICIs can also cause diabetes as a consequence of autoimmune pancreatitis and some cases are characterized by insulin resistance as manifested by a phenotype close to type 2 diabetes or as a decompensation of previously known type 2 diabetes and, less frequently, can be associated with autoimmune generalized lipoatrophy (15).

A genetic component may also contribute as an HLA association has been verified in ICI-induced diabetes. In a systematic review of 91 case reports, susceptible HLA genotypes were present in $61 \%$ and the HLA-DR4, DR3, DR9 and A2 were the dominant HLA serotypes (10). Another retrospective study found also a predominance of HLA-DR 4 in $76 \%$ of patients with ICI-induced diabetes (13) and the dominance of HLA-DR4 suggests an opportunity to identify the patients at highest risk for development of this irAE.

According to European and American guidelines (11,16-19), it is recommended that blood glucose levels are regularly monitored in order to detect diabetes mellitus, that can be either type 1 or type 2 . For example, ASCO guidelines recommend close clinical evaluation for signs and symptoms of new or worsening diabetes, including measuring glucose at baseline, with each treatment cycle during induction for 12 weeks and then every 3-6 weeks thereafter. However, as ICI-induced diabetes resembles fulminant diabetes, even the routine monitorization of blood glucose levels may not detect or predict its occurrence. Indeed, in a retrospective study of 163 melanoma patients treated with anti-PD-1, in which fasting glycemia was collected before, during and after treatment, ICI-induced diabetes occurred in 5 patients; all of these patients had normal pre-treatment serum glucose measurements, neither was detected a drift of glycemia before ketoacidosis onset (20).

The management of auto-immune diabetes is based on insulin therapy and as there is insufficient evidence to suggest that high-dose corticosteroids may reverse ICI-induced diabetes. In addition, systemic steroids will likely worsen glycemic control, therefore they are not 
recommended $(11,16,17)$. The diagnostic work-up generally includes the measurement of C-peptide and autoantibodies against GAD65, islet cell and insulin to distinguish between type 1 and type 2 diabetes. According to ASCO guidelines, patients with grade 1 hyperglycemia (fasting glucose $<160 \mathrm{mg} / \mathrm{dL}$ ) may continue ICI treatment if they are asymptomatic or with mild symptoms and without evidence of ketosis or type 1 diabetes with need for insulin therapy. If the treatment is interrupted, restarting treatment with ICIs may be considered after the patient has achieved glycemic control with insulin therapy and toxicity declines to grade $\leq 1$, only if benefits outweigh risks $(21,22)$.

This case report is consistent with the typical presentation of ICI-induced diabetes, that shares many similarities with fulminant diabetes, in which DKA is often the first manifestation and concomitant thyroid dysfunction is frequently seen. In contrast with other immune-mediated endocrinopathies that are typically of grade $1 / 2$ and are usually detected by routine laboratory testing, autoimmune diabetes can have a rapid onset and cause lifethreatening grade 4 irAE, as described in this case report and previously reported systematic review (10). Therefore, besides monitoring blood glucose levels, patient education regarding this irAE is essential and prompt medical treatment. Screening for susceptibility for auto-immune diabetes and monitoring the blood lipase levels may also be other tools for early detection of this irAE or even avoiding the ICIs in non-metastatic setting if the patient harbor susceptible haplotypes for auto-immune diabetes, but this is not recommended in clinical practice. Further pharmacovigilance studies, including prospective clinical trials and real-world studies, are very important to evaluate the real incidence, severity, and impact on quality of life and overall survival of these irAE in order to better selection of ICI treatment, especially when used in curative setting.

\section{Conclusions}

The occurrence of ICI-induced diabetes highlights the importance of proper selection of patients for ICI treatment, especially in the non-metastatic setting, as this endocrine irAE can be life-threatening and is often irreversible, causing life-long comorbidities and requiring continuous hormone replacement therapy. There is a need for identification of biomarkers associated with increased risk of occurrence of ICI-induced diabetes for selection of patients, as well new biomarkers to allow its early diagnosis and treatment.

\section{Acknowledgments}

Funding: None.

\section{Footnote}

Reporting Checklist: The authors have completed the CARE reporting checklist. Available at http://dx.doi.org/10.21037/ tlcr-20-408

Conflicts of Interest: All authors have completed the ICMJE uniform disclosure form (available at http://dx.doi. org/10.21037/tlcr-20-408). RM reports grants from Merck and Astra Zeneca, personal fees from Bayer outside the submitted work. CR reports grants from MSD, Astra Zeneca, ARCHER, Inivata, Merck Serono, Mylan and Lung Cancer Research Foundation-Pfizer, non-financial support from Oncopass, Guardant Health and Biomark Inc., outside the submitted work. AR reports consultancy/ advisory role for Astra Zeneca, MSD and Roche outside the submitted work. The authors have no other conflicts of interest to declare.

Ethical Statement: The authors are accountable for all aspects of the work in ensuring that questions related to the accuracy or integrity of any part of the work are appropriately investigated and resolved. All procedures performed in studies involving human participants were in accordance with the ethical standards of the institutional and/or national research committee(s) and with the Helsinki Declaration (as revised in 2013). Written informed consent was obtained from the patient for publication of this case report and any accompanying images.

Open Access Statement: This is an Open Access article distributed in accordance with the Creative Commons Attribution-NonCommercial-NoDerivs 4.0 International License (CC BY-NC-ND 4.0), which permits the noncommercial replication and distribution of the article with the strict proviso that no changes or edits are made and the original work is properly cited (including links to both the formal publication through the relevant DOI and the license). See: https://creativecommons.org/licenses/by-nc-nd/4.0/.

\section{References}

1. Antonia SJ, Villegas A, Daniel D, et al. Durvalumab after Chemoradiotherapy in Stage III Non-Small-Cell Lung 
Cancer. N Engl J Med 2017;377:1919-29.

2. Gray JE, Villegas A, Daniel D, et al. Three-year overall survival with durvalumab after chemoradiotherapy in Stage III NSCLC - Update from PACIFIC. J Thorac Oncol 2020;15:288-93.

3. Riley DS, Barber MS, Kienle GS, et al. CARE guidelines for case reports: explanation and elaboration document. J Clin Epidemiol 2017;89:218-35.

4. Brooks ED, Verma V, Senan S, et al. Salvage therapy for locoregional recurrence after stereotactic ablative radiotherapy for early-stage NSCLC. J Thorac Oncol 2020;15:176-89.

5. Sznol M, Postow MA, Davies MJ, et al. Endocrinerelated adverse events associated with immune checkpoint blockade and expert insights on their management. Cancer Treat Rev 2017;58:70-76.

6. Barroso-Sousa R, Barry WT, Garrido-Castro AC, et al. Incidence of endocrine dysfunction following the use of different immune checkpoint inhibitor regimens a systematic review and meta-analysis. JAMA Oncol 2018;4:173-82.

7. FDA Prescribing Information of IMFINZI® (durvalumab). Approval: 2017. Last revised: Available online: 08/2019. https://www.accessdata.fda.gov/ drugsatfda_docs/label/2020/761069s018lbl.pdf

8. Mengíbar JL, Capel I, Bonfill T, et al. Simultaneous onset of type 1 diabetes mellitus and silent thyroiditis under durvalumab treatment. Endocrinol Diabetes Metab Case Rep 2019;2019:10.1530/EDM-19-0045.

9. Wright JJ, Salem JE, Johnson DB, et al. Increased reporting of immune checkpoint inhibitor-associated diabetes. Diabetes Care 2018;41:e150-1.

10. de Filette JMK, Pen JJ, Decoster L, et al. Immune checkpoint inhibitors and type 1 diabetes mellitus: a case report and systematic review. Eur J Endocrinol 2019;181:363-74.

11. Haanen JBAG, Carbonnel F, Robert C, et al. Management of toxicities from immunotherapy: ESMO Clinical Practice Guidelines for diagnosis, treatment and followup. Ann Oncol 2017;28:iv119-iv142.

12. Farina KA, Kane MP. Programmed Cell Death-1 Monoclonal Antibody Therapy and Type 1 Diabetes Mellitus: a Review of the Literature. J Pharm Pract. DOI:10.1177/0897190019850929.

13. Stamatouli AM, Quandt Z, Perdigoto AL, et al. Collateral damage: Insulin-dependent diabetes induced with checkpoint inhibitors. Diabetes 2018;67:1471-80.

14. Michot JM, Ragou P, Carbonnel F, et al. Significance of Immune-related Lipase Increase Induced by Antiprogrammed Death-1 or Death Ligand-1 Antibodies: a Brief Communication. J Immunother 2018;41:84-85.

15. Marchand L, Disse E, Dalle S, et al. The multifaceted nature of diabetes mellitus induced by checkpoint inhibitors. Acta Diabetol 2019;56:1239-45.

16. Higham CE, Olsson-Brown A, Carroll P, et al. Society for endocrinology endocrine emergency guidance: acute management of the endocrine complications of checkpoint inhibitor therapy. Endocr Connect 2018;7:G1-G7.

17. NCCN Clinical Practice Guidelines. Management of Immunotherapy-Related Toxicities, version 2. 2019. Available online: https://www.nccn.org/professionals/ physician_gls/default.aspx

18. Brahmer JR, Lacchetti C, Schneider BJ, et al. Management of immune-related adverse events in patients treated with immune checkpoint inhibitor therapy: american society of clinical oncology clinical practice guideline. J Clin Oncol 2018;36:1714-68.

19. Puzanov I, Diab A, Abdallah K, et al. Managing toxicities associated with immune checkpoint inhibitors: Consensus recommendations from the Society for Immunotherapy of Cancer (SITC) Toxicity Management Working Group. J Immunother Cancer 2017;5:95.

20. Magis Q, Gaudy-Marqueste C, Basire A, et al. Diabetes and Blood Glucose Disorders under Anti-PD1. J Immunother 2018;41:232-40.

21. Edahiro R, Ishijima M, Kurebe H, et al. Continued administration of pembrolizumab for adenocarcinoma of the lung after the onset of fulminant type 1 diabetes mellitus as an immune-related adverse effect: a case report. Thorac Cancer 2019;10:1276-79.

22. Del Rivero J, Cordes LM, Klubo-Gwiezdzinska J, et al. Endocrine-Related Adverse Events Related to Immune Checkpoint Inhibitors: Proposed Algorithms for Management. Oncologist 2020;25:290-300.

Cite this article as: Lopes AR, Russo A, Li AY, McCusker MG, Kroopnick JM, Scilla K, Mehra R, Rolfo C. Development of autoimmune diabetes with severe diabetic ketoacidosis and immunerelated thyroiditis secondary to durvalumab: a case report. Transl Lung Cancer Res 2020;9(5):2149-2156. doi: 10.21037/tlcr-20-408 